\title{
ROTATIONAL VELOCITY DISPERSION IN THE HYADES AND OTHER GALACTIC CLUSTERS FROM THE $V S I N I$ OBSERVATIONS
}

\author{
YVES GAIGÉ \\ Observatoire Midi-Pyrénées
}

\section{INTRODUCTION}

Let us recall briefly the main points of the theory as developped by Chandrasekhar and Münch (1950): if the axis of rotation of a star is inclined with an angle $i$ to the line of sight, the apparent velocity is given by $y=v \sin i$ where $v$ is the true rotational velocity. Assuming that the axes of rotation are randomly distributed in space, the frequency function $f(v)$ of the true velocities may be related to the corresponding $\phi(y)$ of the apparent ones by the integral equations :

$$
\begin{gathered}
\phi(y)=y \int_{y}^{\infty} \frac{f(v)}{v\left(v^{2}-y^{2}\right)^{1 / 2}} d v \\
f(v)=-\frac{2}{\pi} v^{2} \frac{d}{d v} v \int_{v}^{\infty} \frac{\phi(y)}{y^{2}\left(y^{2}-v^{2}\right)^{1 / 2}} d y
\end{gathered}
$$

The central moments of $f(v)$ can be derived directly from the observations by means of (1) without the knowledge of the function itself; for instance, the mean and the mean square deviation are given respectively by :

$$
\langle v\rangle=\frac{4}{\pi}\langle y\rangle \quad\left\langle(v-\langle v\rangle)^{2}\right\rangle=1.5\left\langle y^{2}\right\rangle-\frac{16}{\pi^{2}}\langle y\rangle^{2}
$$

\section{OBSERVED DISTRIBUTIONS}

Apparent distributions have been computed in different colour intervals for the Hyades cluster for which we had new rotational velocities measurements made with CORAVEL and kindly communicated by Mermilliod (1991). The velocities have been expressed in unit of their means.

Since the differentiation of the observed distribution may lead to misleading results, it would be better to assume a form for $f(v)$, work out the corresponding frequency distribution of ( $v \sin i)$, and compare this with the observations. In the present study the model functions (4) have been tested to determine the probable shape of the true distribution.

$$
f_{\text {in }}(v)= \begin{cases}k_{\text {in }}\left(p_{i}^{2}-\left(v-v_{i 0}\right)^{2}\right)^{n} & \text { if } v_{i 0}-p_{i} \leq v \leq v_{i 0}+p_{i} \\ 0 & \text { otherwise }\end{cases}
$$

The corresponding $\phi(y)$ are known analytically, making them suitable forms to try in practical applications. In fact it is hard to decide in favour of one shape. 


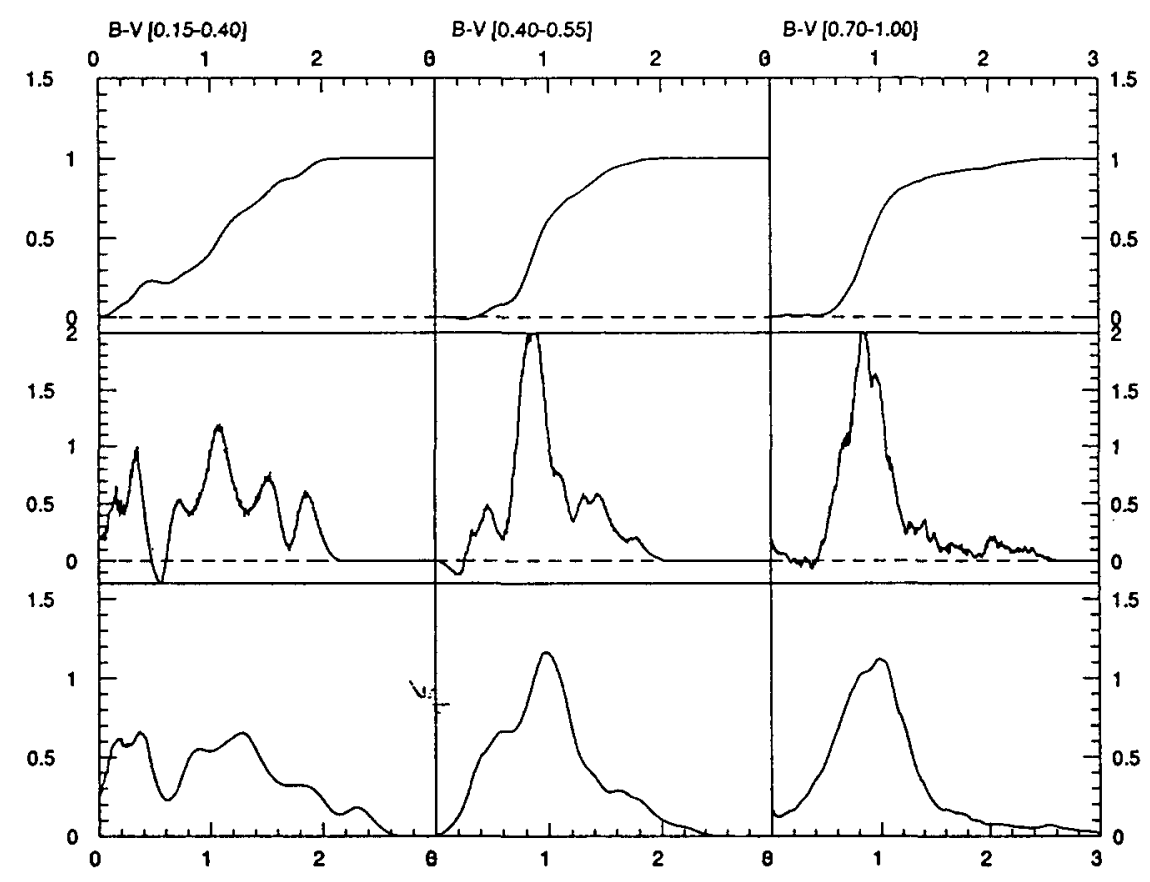

FIGURE I Observed, true and integrated true distributions for the Hyades cluster

$n=0$ gives unrealistic apparent distribution (with an angular point) but is very easy to compute. $n=1$ and $n=2$ are intermediate cases between rectangular and gaussian forms, they give very similar apparent distributions. The advantage of $n=2$ is the continuity of its derivative.

Parameters of the theorical functions may be adjusted by minimizing some distance with the observed distribution, but in the case of unimodale distribution they can be computed directly by determining the variable $\sigma\left(\sigma=\frac{\overline{v^{2}}-\vec{v}^{2}}{\vec{v}^{2}}\right)$ because $\sigma$ is a growing function of $\alpha$ (where $\alpha=\frac{p}{v_{0}}$ ). $\sigma$ is also a growing function of $n$ as $\alpha$ goes to $\infty$.

\section{TRUE DISTRIBUTION}

To get the true distribution we have to use estimators which give a smoother a curve than the histogram, and then do the derivation. This has been done for the Hyades cluster in several colour intervals.

As can be seen on figure (I) the distribution varies with effective temperature. Some peculiar features may be simply explained looking carefully the data : the negative values observed simply show the lack of $v \sin : i$ between 40 and $60 \mathrm{~km} \cdot \mathrm{s}^{-1}$ for $\mathrm{B}-\mathrm{V}=[0.15,0.40]$ and below $20 \mathrm{~km} \cdot \mathrm{s}^{-1}$ for $\mathrm{B}-\mathrm{V}=[0.40,0.55]$. This indicates that in these cases the hypothesis of random orientation for the axis of rotation is not valid : this is probably due to the small number of stars 
in the sample (observational bias).

$\mathrm{B}-\mathrm{V}=[0.15,0.40]$ the distribution was often assumed to be uniform, which is not exactly the case with the actual measurements.

$\mathrm{B}-\mathrm{V}=[0.40,0.55]$ this distribution is well described by the sum of two distribution of the same importance, a widely spread one and a sharper one.

$\mathrm{B}-\mathrm{V}=[0.70,1.00]$ the distribution is very similar to the precedent : at the same location, there is a slightly wider peak and there is a very faint but long wing towards high velocities.

\section{INTEGRATED TRUE DISTRIBUTION}

In order to get the integrated distribution, let us integrate the relation (2) between 0 and $z$. After reduction the equation becomes :

$$
\int_{0}^{z} f(v) d v=\int_{0}^{z} \phi(y) d y+\frac{2}{\pi} \int_{z}^{\infty} \phi(y) \frac{d}{d y}\left(y \arcsin \left(\frac{z}{y}\right)\right) d y
$$

It is then possible to compute the integrated distribution of the true rotational velocity with only knowing the apparent distribution $\phi(y)$, without any problems of derivation. Computations have been done for the Hyades cluster for the same colour intervals.

\section{CONCLUDING REMARKS}

In section (2) we study the rotational behaviour of a group of stars, by fitting the apparent distribution with some model functions. It has been found that in general the observed distribution can not be simply fitted by an unimodale distribution. This observed distribution varies greatly with colour.

- above $7000 \mathrm{~K}$ the velocity distribution is almost uniform

- between 6400 and $7000 K$ the distribution is roughly bimodale.

It has then been shown (section (3)) that the distribution of true rotational velocities may be directly computed if the observed distribution is known with an estimator giving a curve smooth enough to do the derivation. This method confirms the fact that the distribution presents one or two peaks (mainly) below $7000 \mathrm{~K}$ whereas above the uniformity is questionnable, probably because of the size of the sample.

Finally, we made an improvment of the theory, by computing the integrated true distribution from the observed one without any free parameter or assumption. Contrary to the inverse problem this method can be used whatever the estimator.

\section{REFERENCES}

Chandrasekhar, S., and Münch, G. 1950 Ap. J., 111, 142.

Mermilliod, J. C. 1991 Private Communication 